\title{
Immune Intervention in Type 1 Diabetes
}

\author{
Bimota Nambam ${ }^{1}$, Natasa Bratina ${ }^{2}$, Desmond Schatz ${ }^{1}$
}

Introduction

M ULTIPLE STUDIES OVER THE PAST DECADE have reported residual $\beta$-cell function in early type 1 diabetes (T1D), which can last many years after diagnosis. Maintenance of residual endogenous insulin secretion is beneficial in achieving better glycemic control, and hence decreasing the risk of micro- and macrovascular complications. Although the etiopathogenesis of T1D remains to be determined, it is clear that derangements in the immune system play a vital role in the loss of tolerance and subsequent $\beta$-cell destruction. Several attempts have been made to interdict the disease at different stages of disease development. These include primary (before the development of islet autoantibodies) and secondary prevention studies (islet autoantibody positive but before the development of clinical diabetes) as well as in newonset patients and, more recently, in patients with established diabetes. Agents that have been studied in the past few years include oral insulin, GAD vaccine, DiaPep277, anti-CD3, anti-CD20, CTLA-4 IgG, anti-TNF, IL-2, and others. Although the disease has yet to be prevented or reversed, some studies have shown at least transient preservation of $\beta$-cell function. Given the complex interplay between the multiple immune pathways in the etiopathogenesis of T1D, and the lack of understanding of the precise mechanisms culminating in the disease, it is not surprising that we have yet to be able to prevent and cure the disease. However, it is becoming clearer that a combination of therapies and approaches will become more of the future norm. This article reviews some of the key immune interventional and mechanistic studies in T1D subjects that have been published (in print) between July 2014 and June 2015.

\section{Key Articles Reviewed for this Article}

Effects of high-dose oral insulin on immune responses in children at high risk for type 1 diabetes. The pre-POINT randomized clinical trial

Bonifacio $E^{1,2,3}$, Ziegler $A G^{3,4}$, Klingensmith $G^{5}$, Schober $E^{6}$, Bingley $P^{7}$, Rottenkolber $M^{8}$, Theil $A^{1}$, Eugster $A^{1}$, Puff $R^{4}$, Peplow $C^{4}$, Buettner $F^{9}$, Lange $K^{10}$, Hasford $J^{8}$, Achenbach $P^{3,4}$, for the Pre-POINT Study Group

JAMA 2015; 313: 1541-49

Anti-thymocyte globulin/G-CSF treatment preserves $\beta$-cell function in patients with established type 1 diabetes

Haller $M J^{1}$, Gitelman $S E^{2}$, Gottlieb $P A^{3}$, Michels $A W^{3}$, Rosenthal $S M^{2}$, Shuster $J^{4}$, Zou $B^{4}$, Brusko $T M^{5}$, Hulme $M A^{5}$, Wasserfall $C A^{5}$, Mathews $C E^{5}$, Atkinson $M A^{5}$, Schatz $D A^{l}$

J Clin Invest 2015; 125: 448-55

\footnotetext{
${ }^{1}$ Department of Pediatrics, Division of Endocrinology, University of Florida, Gainesville, FL

${ }^{2}$ Department of Endocrinology, Diabetes and Metabolic Diseases, University Medical Centre, University Childrens Hospital Ljubljana, Ljubljana, Slovenia
} 


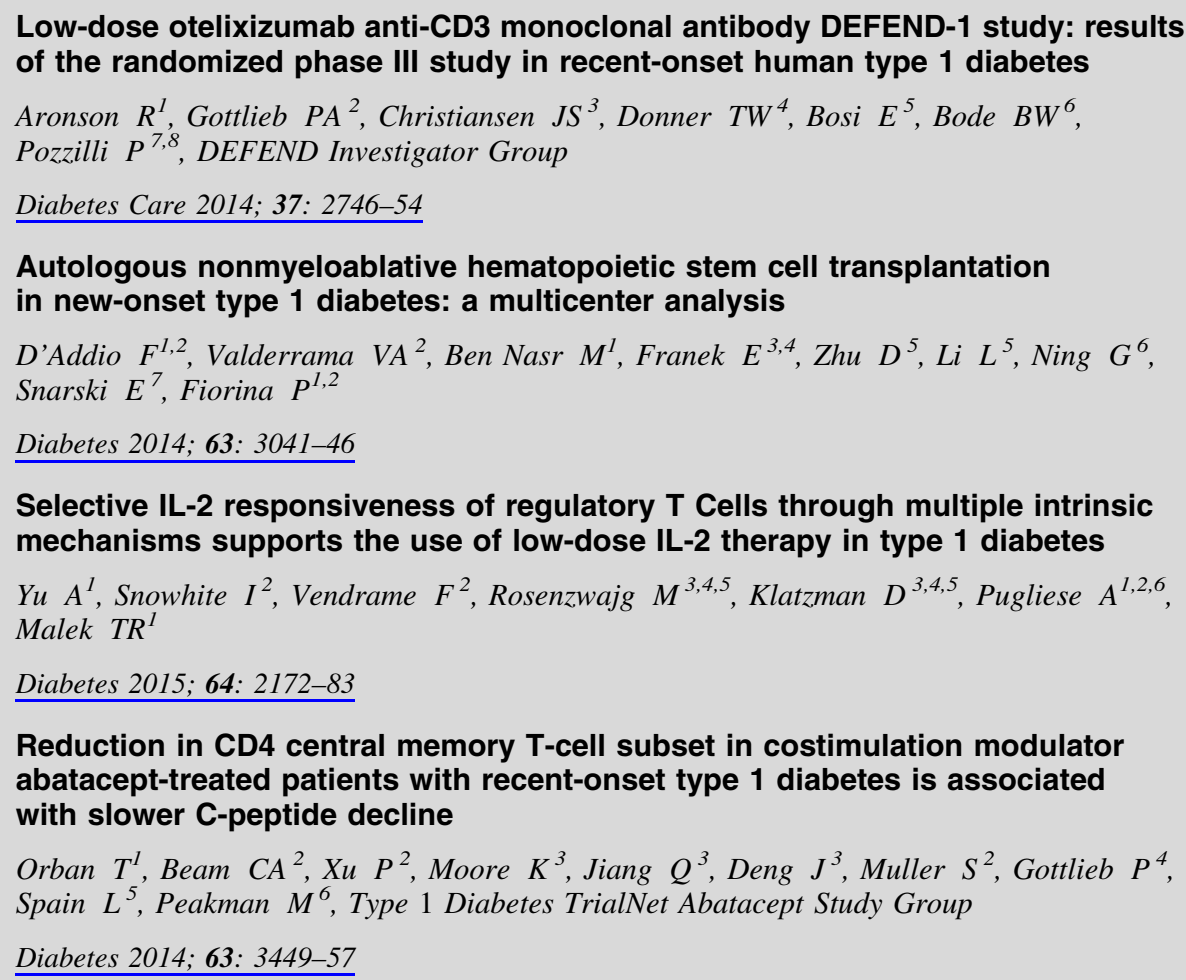

\section{Effects of high-dose oral insulin on immune re- sponses in children at high risk for type 1 diabetes. The pre-POINT randomized clinical trial}

Bonifacio $E^{1,2,3}$, Ziegler $A G^{3,4}$, Klingensmith $G^{5}$, Schober $E^{6}$, Bingley $\mathrm{PJ}^{7}$, Rottenkolber $M^{8}$, Theil $A^{1}$, Eugster $A^{1}$, Puff $R^{4}$, Peplow $C^{4}$, Buettner $F^{9}$, Lange $K^{10}$, Hasford $J^{8}$, Achenbach $P^{3,4}$, for the Pre-POINT Study Group

${ }^{1}$ DFG Center for Regenerative Therapies Dresden, Faculty of Medicine, Technische Universität Dresden, Dresden, Germany; ${ }^{2}$ Paul Langerhans Institute Dresden, German Center for Diabetes Research (DZD), Technische Universität Dresden, Dresden, Germany; ${ }^{3}$ Forschergruppe Diabetes e.V., Neuherberg, Germany; ${ }^{4}$ Institute of Diabetes Research, Helmholtz Zentrum München, and Forschergruppe Diabetes, Klinikum rechts der Isar, Technische Universität München, Munich, Germany; ${ }^{5}$ Barbara Davis Center for Childhood Diabetes, University of Colorado School of Medicine, Aurora, CO; ${ }^{6} \mathrm{De}$ partment of Pediatrics, Medical University of Vienna, Vienna, Austria; ${ }^{7}$ School of Clinical Sciences, University of Bristol, Bristol, UK; ${ }^{8}$ Institute for Medical Information Sciences, Biometry, and Epidemiology, Ludwig-Maximilians-Universität, Munich, Germany; and ${ }^{9}$ Institute of Computational Biology, Helmholtz Zentrum München; ${ }^{10}$ Department of Medical Psychology, Hannover Medical School, Hannover, Germany

JAMA 2015; 313: 1541-49

This manuscript is also discussed in the article on: Diabetes Technology and Therapy in the Pediatric Age Group, p. S-97.

\section{Background}

The Diabetes Prevention Trial-Type 1 (DPT-1) demonstrated a possible protective effect of oral insulin $(7.5 \mathrm{mg})$ in subgroups at risk for T1D (IAA > $>80 \mathrm{nU} / \mathrm{mL}$ ) as compared to placebo (annualized T1D rate was $6.2 \%$ vs $10.4 \%, P=0.015$ ). An even greater protective effect was noted in those with even higher (IAA > $300 \mathrm{nU} / \mathrm{ml}$ ) titers. After cessation or oral insulin, this subgroup had a delay in T1D development by 2.2 years (1). The precise mechanisms for this immunoprotective effect have yet to be elucidated but has been hypothesized to be secondary to induction of antigen-specific T-regulatory cells. This pilot study explored the immunoprotective effects of escalating doses of daily oral insulin, in genetically at-risk, antibody negative children.

\section{Methods}

This was a randomized, double-blinded, placebo-controlled, phase 2 pilot study in first degree relatives aged 2-7 years, with T1D high-risk HLA class-II genes and who were confirmed to be islet autoantibody-negative. Children were randomized to receive oral insulin $(n=15)$ or placebo $(n=10)$ once daily for 3 to 18 months. Nine children received insulin with dose escalations from 2.5 to $7.5 \mathrm{mg}(n=3), 2.5$ to $22.5 \mathrm{mg}(n=3)$, or 7.5 to $67.5 \mathrm{mg}(n=3)$ after 6 months; 6 children only received doses of $22.5 \mathrm{mg}(n=3)$ or $67.5 \mathrm{mg}(n=3)$.

\section{Results}

Increase in serum $\operatorname{IgG}$ and salivary $\operatorname{IgA}$ binding to insulin, and $\mathrm{CD}^{+}{ }^{+} \mathrm{T}$-cell proliferative responses to insulin were observed in 2/10 placebo-treated children, 1/6 children treated with $2.5 \mathrm{mg}$ of insulin, $1 / 6$ treated with $7.5 \mathrm{mg}, 2 / 6$ treated with $22.5 \mathrm{mg}$, and $5 / 6$ treated with $67.5 \mathrm{mg}(P=0.02)$. Insulin-responsive $\mathrm{T}$-cells displayed regulatory $\mathrm{T}$-cell features after oral insulin treatment. No hypoglycemia, IgE responses to insulin, or islet autoantibodies were observed. 


\section{Conclusion}

Higher-dose daily oral insulin administration showed immunoprotective effects in children genetically at high risk for T1D.

\section{Comment}

This pilot study demonstrates that high doses of oral insulin may have protective immunomodulatory effects in those genetically at risk for T1D. Whether such therapy will potentially prevent seroconversion and progression to T1D needs to be determined with longer follow-up. Although no adverse events were attributed to the administration of oral insulin, the sample size was small and the follow-up duration was short. The data are encouraging and will need to be confirmed in other larger studies. Future studies should also include children younger than 2 years (children in this study were $>2$ years) as peak seroconversion occurs between 9 months and 2 years. Bonifacio et al. only studied children with a higher genetic risk for T1D ( $<1 \%$ of children who develop T1D), and the immune response of high dose oral insulin may be different in those with a lower genetic risk. It will also be interesting to determine whether high dose oral insulin would be beneficial to subjects who have already seroconverted. Other antigen-based therapies include a phase $1 \mathrm{~b}$ trial using proinsulin peptides (2); this was recently completed July 2015 with a phase 2 study expected to begin in 2016. In addition, combinations including the coadministration of other autoantigens (e.g., proinsulin or glutamic acid decarboxylase) along with other immunomodulatory agents such as antiCD3 monoclonal antibodies or anti-thymocyte globulin (ATG), and granulocyte colony stimulating factor (GCSF) are also being investigated.

\section{Anti-thymocyte globulin/G-CSF treatment pre- serves $\beta$-cell function in patients with established type 1 diabetes}

Haller $M J^{1}$, Gitelman $S E^{2}$, Gottlieb $P A^{3}$, Michels $A W^{3}$, Rosenthal $\mathrm{SM}^{2}$, Shuster $\mathrm{JJ}^{4}$, Zou $\mathrm{B}^{4}$, Brusko $\mathrm{TM}^{5}$, Hulme $M A^{5}$, Wasserfall $C A^{5}$, Mathews $C E^{5}$, Atkinson $M A^{5}$, Schatz $D A^{1}$

${ }^{I}$ Department of Pediatrics, Division of Endocrinology, University of Florida, Gainesville, $F L ;{ }^{2}$ Department of Pediatrics, Division of Endocrinology, UCSF, San Francisco, CA; ${ }^{3}$ Department of Pediatrics and Medicine, Division of Endocrinology, University of Colorado, Denver, CO; ${ }^{4} \mathrm{De}$ partment of Biostatistics, University of Florida, College of Medicine, Gainesville, FL; ${ }^{5}$ Department of Pathology, University of Florida, College of Medicine, Gainesville, FL

\section{J Clin Invest 2015; 125: 448-55}

\section{Background}

Previous efforts to preserve $\beta$-cell function in individuals with T1D have mainly focused on single immunomodulatory agents administered within 100 days of diagnosis. Based on promising preclinical studies in NOD mice and humans, the authors hypothesized that combination low-dose anti- thymocyte globulin (ATG) and pegylated granulocyte CSF (G-CSF) would preserve $\beta$-cell function in patients with established T1D (duration of T1D $>4$ months and $<2$ years).

\section{Methods}

This was a randomized, single-blinded, placebo-controlled, phase-2 study in subjects $12-45$ years with T1D duration $>4$ months to $<2$ years; 17 subjects were administered low dose ATG and peg G-CSF as per study protocol while 8 subjects received placebo. The 1-year post-treatment AUC C-peptide (4-hour mixed-meal tolerance test/MMTT) was assessed along with change in insulin use, A1c, and immune markers.

\section{Results}

MMTT-stimulated AUC C-peptide at 12 months post therapy, was significantly higher in the study group compared to placebo group $(P=0.017)$; the majority of the subjects in the treatment group also showed nondeclining beta cell function even after 12 months. The study group also had significantly higher Tregs compared to placebo at 2 weeks and 12 months.

\section{Conclusions}

Combination immunotherapy with ATG and G-CSF preserved $\beta$-cell function in T1D patients with disease duration $<2$ years.

\section{Comment}

Autologous nonmyeloablative stem cell transplantation using a regimen of cyclophosphamide, granulocyte CSF (G-CSF), autologous stem cell harvest and infusion after conditioning with ATG plus cyclophosphamide, and intensive inpatient as well as outpatient support, demonstrated the capacity to sustain $\beta$ cell function and achieve short-term insulin independence (3). However, performing such a study has raised questions about equipoise. Haller et al. used a "Brazil-lite" cocktail, and although there were a number of side effects seen in the experimental group, including cytokine-release syndrome and serum sickness, these were transient and fully reversible and not major adverse effects. Unlike other new-onset studies enrolling patients within 100 days, the authors enrolled subjects with duration ranging from 4 to 24 months and after 12 months of follow-up, $\beta$-cell function was retained at baseline levels in 9 of 16 subjects in the treated group. Interestingly, ATG and G-CSF together demonstrate preservation of $\beta$-cell function in T1D whereas neither preserve $\beta$-cell function when used alone (4,5). Mechanistically, unlike high-dose ATG, the low dose ATG and G-CSF combination induces a less severe T-cell depletion, allows for faster T-cell recovery, and induces Tregs. It should be emphasized that this study was small (only 25 subjects, randomized 2:1), and there were some baseline differences between experimental and placebo groups. A larger clinical study on the efficacy of ATG and G-CSF in new-onset T1D $(<3$ months post diagnosis) patients is currently being conducted in TrialNet (6). It will be interesting, too, to determine whether redosing might be required for ongoing 
preservation of $\beta$ cell function. The next step is to determine whether the administration of ATG-GCSF at an even earlier stage, that is, in high-risk, pre-T1D subjects, will prevent or delay the onset of the disease.

\section{Low-dose otelixizumab anti-CD3 monoclonal anti- body DEFEND-1 study: results of the randomized phase III study in recent-onset human type 1 diabetes}

Aronson $R^{1}$, Gottlieb $P A^{2}$, Christiansen $J S^{3}$, Donner $T W^{4}$, Bosi $E^{5}$, Bode $B W^{6}$, Pozzilli $P^{7,8}$, DEFEND Investigator Group

${ }^{1}$ LMC Diabetes and Endocrinology, Toronto, Ontario, Canada; ${ }^{2}$ University of Colorado, Denver, CO; ${ }^{3}$ University of Aarhus, Aarhus University Hospital, Aarhus, Denmark; ${ }^{4}$ Johns Hopkins University School of Medicine, Baltimore, MD; ${ }^{5}$ San Raffaele Hospital Scientific Institute, Vita Salute San Raffaele University, Milan, Italy; ${ }^{6}$ Emory University, Atlanta Diabetes Associates, Atlanta, GA; ${ }^{7}$ Università Campus Bio-Medico di Roma, Rome, Italy; and ${ }^{8}$ Barts and the London School of Medicine and Dentistry, Queen Mary, University of London, London, $U K$

Diabetes Care 2014; 37: 2746-54

\section{Background}

Previous animal and human studies have demonstrated the $\beta$ cell-preserving effects of otelixizumab, a chimeric monoclonal antibody targeting the CD3/T-cell receptor. Trials evaluating higher doses of otelixizumab $(48-64 \mathrm{mg}$ ) had reported higher EBV infection reactivation. In this study, the authors have attempted to evaluate the immunomodulatory effects of a low-dose regimen in new onset T1D subjects.

\section{Methods}

This was a randomized, placebo-controlled, multinational trial with 272 T1D patients $(12-45$ years, $<90$ days between diagnosis and first dose) randomized to treatment with $3.1 \mathrm{mg}$ otelixizumab or placebo. Change in C-peptide area under the curve (AUC) after a 2-h mixed-meal tolerance test at month 12 was assessed.

\section{Results}

There was no difference in the change from baseline in 2 hour C-peptide between otelixizumab and the placebo group at 12 months. Differences in Tregs level, A1c, and insulin dosing between the two groups were not demonstrated. EBV infections were not observed in the study group.

\section{Conclusion}

Low dose otelixizumab was tolerated better by patients but failed to preserve $\beta$-cell function or improve metabolic control.

\section{Comment}

This study utilized a much lower dose of otelixizumab (3.1 mg) compared to the Belgian Phase II Registry study where the total dose was 48-64 mg (7). It is not precisely clear why this particular dose was chosen other than to target a lower rate of EBV reactivation ( $75 \%$ was seen in the phase II trial). Transient reductions in lymphocytes and modulation of CD3 T-cell receptors were observed; however, the maximum reduction in lymphocytes was less than that observed during therapy in the phase II trial using otelixizumab and in previous trials with teplizumab (8), another anti-CD3 monoclonal antibody. At a dose of $3.1 \mathrm{mg}$, no efficacy was seen, although symptoms of cytokine release syndrome were significantly milder, and no reactivation of EBV was seen. It is clear that further dose finding in new-onset T1D patients are required to establish the efficacy of anti-CD3 studies together with a tolerable safety profile. A study in TrialNet is currently ongoing to evaluate whether teplizumab can prevent or delay progression to clinical disease in highrisk, islet autoantibody positive relatives with glucose intolerance (9). It will be interesting to see whether using it in combination with other agents such as oral insulin and/or G-CSF would enhance efficacy while keeping side effects to a minimum.

\section{Autologous nonmyeloablative hematopoietic stem cell transplantation in new-onset type 1 diabetes: a multicenter analysis}

D'Addio $F^{1,2}$, Valderrama $V A^{2}$, Ben Nasr $M^{1}$, Franek $E^{3,4}$, Zhu $D^{5}$, Li $L^{5}$, Ning $G^{6}$, Snarski $E^{6}$, Fiorina $P^{1,2}$

${ }^{1}$ Nephrology Division, Boston Children's Hospital, Harvard Medical School, Boston, MA; ${ }^{2}$ Transplant Medicine, IRCCS San Raffaele Hospital, Milan, Italy; ${ }^{3}$ Department of Internal Diseases, Diabetology and Endocrinology, Central Hospital, Ministry of Interior Affairs and Administration, Warsaw, Poland, and Department of Endocrinology, Mossakowski Medical Research Centre, Polish Academy of Sciences, Warsaw, Poland; ${ }^{4}$ Division of Endocrinology, The Affiliated Drum Tower Hospital of Nanjing University, Nanjing, Jiangsu, People's Republic of China; ${ }^{5}$ Shanghai Jiao Tong University School of Medicine, Shanghai, People's Republic of China; ${ }^{6}$ Department of Hematology, Oncology and Internal Diseases, Medical University of Warsaw, Warsaw, Poland

\section{Diabetes 2014; 63: 3041-46}

\section{Background}

Independence from exogenous insulin in early T1D patients following immunoablation and reconstitution of the immune system with autologous stem cell transplantation have been demonstrated in earlier studies.

\section{Methods}

In all, 65 individuals, 12-35 years, with T1D duration up to 6 weeks (Poland)-12 months (China), were recruited. Cyclophosphamide, ATG, and G-CSF were used for conditioning immunosuppression therapy followed by autologous hematopoietic stem cell (HSC) infusion. The Polish cohort 
received plasmapheresis before HSC infusion. Subjects were followed for 48 months.

\section{Results}

Insulin independence was observed in 59\% of treated individuals within the first 6 months and 32\% still remained insulin independent at 48 months. C- peptide levels were increased from baseline at 6 months and maintained at 48 months post treatment. Serious adverse effects were frequent. Poor responders were younger at the time of enrollment, were treated later after T1D diagnosis, had more instances of DKA, and had higher HbA1c levels at the time of study enrollment.

\section{Conclusions}

Autologous nonmyeloablative HSC transplantation has the potential to induce remission in selected T1D individuals. However, the serious adverse event profile limits clinical implementation.

\section{Comment}

$\beta$-cell preservation for up to 4 years was seen in the majority of patients (those with T1D of short duration) post therapy. However, the frequent occurrence of serious adverse events (over 50\%) and death (in one subject) raises serious ethical concerns about furthering studies under this protocol. Whether the observed effects were due to the heavy immunosuppression regimen prior to autologous HSC transplantation, the HSC transplantation itself, or both, needs to be determined. The authors further divided the treated group into good and poor responders based on insulin independence at 6 months. Though the poor responders were characterized (younger at enrollment, treated later after T1D diagnosis, had more instances of DKA, and had higher HbA1c levels at the time of study enrollment), it is not clear what role, if any, plasmapheresis may have played. Although the authors have made favorable comparison to outcomes following whole pancreas transplantation (and superiority over islet transplantation), whole organ transplantation (e.g., simultaneous pancreatic-kidney, pancreasafter-kidney) is invariably reserved for those with significant complications.

\section{Selective IL-2 responsiveness of regulatory T Cells through multiple intrinsic mechanisms supports the use of low-dose IL-2 therapy in type 1 diabetes}

$Y u A^{I}$, Snowhite $I^{2}$, Vendrame $F^{2}$, Rosenzwajg $M^{3,4,5}$, Klatzman $D^{3,4,5}$, Pugliese $A^{1,2,6}$, Malek $T R^{1}$

${ }^{I}$ Department of Microbiology and Immunology, Miller School of Medicine, University of Miami, Miami, FL; ${ }^{2}$ Diabetes Research Institute, Miller School of Medicine, University of Miami, Miami, FL; ${ }^{3}$ Assistance Publique-Hôpitaux de Paris, Hôpital Pitié-Salpêtrière, Biotherapy (CIC-BTi) and Inflammation-Immunopathology-Biotherapy Department (I2B), Paris, France; ${ }^{4}$ Sorbonne Université, Université Pierre et Marie Curie Univ Paris 06, Unité Mixte de Recherche (UMR)-S 959, Immunology-Immunopathology-Immunotherapy (I3), Paris, France; ${ }^{5}$ INSERM, UMR-S 959, Immunology-
Immunopathology-Immunotherapy (I3), Paris, France; ${ }^{6}$ Department of Medicine, Miller School of Medicine, University of Miami, Miami, FL

Diabetes 2015; 64: 2172-83

\section{Background}

Low-dose IL-2 administration has been shown to increase Tregs; Tregs induce tolerogenesis and could potentially suppress islet cell autoimmunity. Low-dose IL-2 therapy has been and is currently being investigated in clinical trials in established T1D patients. The authors of this study have attempted to define the mechanisms leading to the preferential induction of Tregs with low dose IL-2 and the rationale for this approach in T1D.

\section{Methods}

Mechanistic in vitro and in vivo studies were performed using peripheral blood samples from established T1D patients and healthy controls.

\section{Results}

IL-2-dependent STAT5 activation in Tregs from healthy individuals and patients with T1D occurred at an $\sim 10$-fold lower concentration of IL-2 than that required by T memory $\left(\mathrm{T}_{\mathrm{M}}\right)$ cells or by in vitro-activated T-cells. This selective Treg responsiveness is explained by their higher expression of IL-2 receptor subunit $\alpha$ (IL-2R $\alpha)$ and $\gamma$ chain and also endogenous serine/threonine phosphatase protein phosphates 1 and/or 2A activity. Genome-wide profiling identified an IL2-dependent transcriptome in human Tregs. Quantitative assessment of selected targets indicated that most were optimally activated by a 100 -fold lower concentration of IL-2 in Tregs versus $\mathrm{CD} 4^{+} \mathrm{T}_{\mathrm{M}}$ cells.

\section{Conclusion}

Immunotolerance observed with low dose IL-2 may be mediated by a set of specific conditions that converge to selectively support the activation and function of Tregs.

\section{Comment}

Depletion of Treg cells leads to inflammation and multiorgan autoimmune disease, which likely results from an imbalance in regulatory and effector cells. There has been a lot of interest in developing protocols that boost Treg number and function in T1D. A previous study that utilized higher doses of IL-2 together with rapamycin demonstrated increased Tregs but worsening of $\beta$-cell function in early T1D. Whether this was due to the deleterious effects of rapamycin on $\beta$-cells or the immune system is not determined (10). Low-dose IL-2 has been demonstrated to specifically activate and expand the Treg cell population, which is likely critical in sustaining immunological tolerance. However, as both T effector cells and NK cells also respond to IL-2 (albeit at higher doses), it is critical to determine whether lower doses induce targeted increases in Tregs. What remains to be determined is not only whether this low dose will be safe and efficacious, but also, and most critically, what precise roles Tregs and IL-2 play in the pathogenesis of T1D. 


\section{Reduction in CD4 central memory T-cell subset in costimulation modulator abatacept-treated patients with recent-onset type 1 diabetes is associated with slower C-peptide decline}

Orban $T^{l}$, Beam $\mathrm{CA}^{2}, \mathrm{Xu} P^{2}$, Moore $K^{3}$, Jiang $Q^{3}$, Deng $J^{3}$, Muller $S^{2}$, Gottlieb $P^{4}$, Spain $L^{5}$, Peakman $M^{6}$, Type 1 Diabetes TrialNet Abatacept Study Group

${ }^{1}$ Joslin Diabetes Center, Boston, MA; ${ }^{2}$ Division of Informatics and Biostatistics, Department of Pediatrics, University of South Florida, Tampa, FL; ${ }^{3}$ David H. Murdock Research Institute, Kannapolis, $N C ;{ }^{4}$ University of Colorado Barbara Davis Center for Childhood Diabetes, Aurora, CO; ${ }^{5}$ National Institute of Diabetes and Digestive and Kidney Diseases, National Institutes of Health, Bethesda, MD; ${ }^{6}$ Peter Gorer Department of Immunobiology, School of Medicine, King's College London, London, United Kingdom

Diabetes 2014; 63: 3449-57

\section{Background}

The authors had previously reported that abatacept treatment for 24 months, administered to recent onset T1D subjects, significantly reduces the decline of $\beta$-cell function (11). The investigators sought to determine the potential mechanism by exploring changes in peripheral blood immune cell subsets after treatment. Possible novel biomarkers that might predict C-peptide changes were also sought.

\section{Methods}

New-onset T1D patients between 6-45 years, disease duration $<100$ days, and stimulated C-peptide levels $>0.2 \mathrm{pmol} /$ $\mathrm{mL}$ were randomized. A total of 77 subjects received abatacept and 35 received placebo. Abatacept treatment was continued till month 24 after initiation. Peripheral blood immune cell immunophenotyping was performed using flow cytometry at baseline, 3, 6, 12, 24, and 30 months following therapy and correlated with C-peptide.

\section{Results}

An increase in circulating $\mathrm{CD} 45 \mathrm{R} 0{ }^{+} \mathrm{CD} 6 \mathrm{~L}^{+} \mathrm{CD} 4$ central memory $\mathrm{T}$-cells was associated with a faster decline in C-peptide levels in the placebo group. In contrast, expansion of naive (CD45R0 $\left.{ }^{-} \mathrm{CD}^{2} 2 \mathrm{~L}^{+}\right) \mathrm{CD} 4 \mathrm{~T}$-cells and a decline in CD45R0 ${ }^{+} \mathrm{CD}_{62} \mathrm{~L}^{+} \mathrm{CD} 4 \mathrm{~T}$-cells was seen in the abatacept-treated group, which was associated with a significantly slower rate of C-peptide decline. This finding was no longer observed 6 months after the last abatacept treatment. Also, an increase in the number of circulating $\mathrm{CD}^{+}$ $\mathrm{T}$-cells and $\mathrm{CD}^{+}$/naive $\mathrm{CD} 4 \mathrm{~T}$-cells ratio during a preceding visit were significantly associated with subsequent decline in C-peptide values, which was altered by abatacept treatment.

\section{Conclusion}

Costimulation blockade with abatacept decreases CD45R $0^{+}$ $\mathrm{CD} 62 \mathrm{~L}^{+} \mathrm{CD} 4^{+} \mathrm{T}$-cells, which is associated with a decrease in C-peptide decline.

\section{Comment}

Novel and valid biomarkers of both $\beta$-cell health and decline, the identification of responders as well as of the autoimmune process itself culminating in T1D are critically needed. Orban et al. previously reported that continuous 24-month costimulation blockade by abatacept significantly slows the decline of $\beta$-cell function after diagnosis (11). They subsequently evaluated peripheral blood immune cell subsets $(\mathrm{CD} 4, \mathrm{CD} 8$-naive, memory and activated subsets, myeloid and plasmacytoid dendritic cells, monocytes, B lymphocytes, $\mathrm{CD}^{+}$ $\mathrm{CD} 25^{\text {high }}$ regulatory T-cells, and invariant NK T-cells) by flow cytometry at baseline, $3,6,12,24$, and 30 months after treatment and observed the expansion of naive $\left(\mathrm{CD} 45 \mathrm{R}^{-} \mathrm{CD}^{-} \mathrm{L}^{+}\right) \mathrm{CD} 4 \mathrm{~T}$-cells in association with a significantly slower rate of C-peptide decline. Interestingly, in the placebo group, an increase in central memory (CM) CD4 T-cells (CD4 ${ }^{+} \mathrm{CD} 45 \mathrm{R} 0^{+} \mathrm{CD} 2 \mathrm{~L}^{+}$) was significantly associated with $\mathrm{C}$-peptide decline at the subsequent visit. Although these are among the first immunological biomarkers correlating with C-peptide level decline in patients with T1D, what remains to be determined are (1) the functional significance of such changes, (2) whether these observed changes and correlation with C-peptide loss will be observed with other interventions and therefore prove to be reliable biomarkers, and (3) whether reversing these findings may alter the course of the disease. Clearly, more validation studies are required.

\section{Conclusion}

In the past 5 years, more than 15 intervention studies at different stages of the disease process have been reported. Where possible benefit has been demonstrated, the therapies have been expanded to studies in larger or different populations. These include primary prevention studies (TRIGR) and secondary prevention trials (TrialNet oral insulin, teplizumab, and abatacept studies) while the remainder are in recent onset T1D subjects. The majority of these studies employ single immunomodulatory agents. Based on experience with other autoimmune diseases, cancer, and some of the studies reported herein, a combination of immunomodulatory agents is mostly likely to interdict the disease process. A better understanding of the precise mechanisms leading to the disease is critical to the development of better therapies. Hopefully studies such as The Environmental Determinants of Diabetes in the Young (TEDDY) and Network for Pancreatic Organ Donors (nPOD) will soon enlighten such mechanisms.

\section{Author Disclosure Statement}

No competing financial interests exist for B.M., N.B., and D.S.

\section{References}

1. Vehik K, Cuthberson D, Ruhlig H, et al. Long-term outcome of individuals treated with oral insulin. Diabetes Prevention Trial-Type 1 (DPT-1) oral insulin trial. Diabetes Care 2011; 34: 1585-90. 
2. Cardiff University. Safety study to assess whether proinsulin peptide injections can slow or stop the body damaging its own insulin making cells in the pancreas in patients newly diagnosed with type 1 diabetes (MonoPepT1De) [article online]. Available at https://clinicaltrials.gov/ct2/show/ NCT01536431 NLM Identifier: NCT01536431 (accessed July 2015).

3. Voltarelli JC, Couri CEB, Stracieri ABPL, et al. Autologous nonmyeloablative hematopoietic stem cell transplantation in newly diagnosed type 1 diabetes mellitus. JAMA 2007; 297: 1568-76.

4. Haller MJH, Cintron M, McGrail K, Brusko TM, Wasserfall C, Hulme M, Mathews CE, Atkinson MA, Schatz DA. Granulocyte colony stimulating factor (GCSF) fails to preserve beta-cell function in patients with recent onset type 1 diabetes (T1D). Diabetes 2014; 63: A428.

5. Gitelman SE, Gottlieb PA, Rigby MR, et al. Antithymocyte globulin treatment for patients with recent-onset type 1 diabetes: 12-month results of a randomised, placebocontrolled, phase 2 trial. Lancet Diabetes Endocrinol 2013; 1: $306-16$.

6. National Institute of Diabetes and Digestive and Kidney Diseases. ATG-GCSF in new onset type 1 diabetes [article online]. Available at https://clinicaltrials.gov/ct2/show/
NCT02215200 NLM Identifier: NCT02215200 (accessed July 2015).

7. Keymeulen B, Vandemeulebroucke E, Ziegler AG. Insulin needs after CD3-antibody therapy in new-onset type 1 diabetes. N Engl J Med 2005; 352: 2598-608.

8. Herold KC, Gitelman S, Greenbaum C, et al. Treatment of patients with new onset Type 1 diabetes with a single course of anti-CD3 mAb Teplizumab preserves insulin production for up to 5 years. Clin Immunol 2009; 132: 166-73.

9. National Institute of Diabetes and Digestive and Kidney Diseases. Teplizumab for prevention of type 1 diabetes in relatives "at-risk" [article online]. Available from https:// clinicaltrials.gov/ct2/show/NCT01030861 NLM Identifier: NCT01030861 (accessed July 2015).

10. Long SA, Rieck M, Sanda S, et al. Diabetes TrialNet and the Immune Tolerance Network. Rapamycin/IL-2 combination therapy in patients with type 1 diabetes augments Tregs yet transiently impairs $\beta$-cell function. Diabetes 2012; 61: 2340-48.

11. Orban T, Bundy B, Becker DJ, et al. Costimulation modulation with abatacept in patients with recent-onset type 1 diabetes: follow-up 1 year after cessation of treatment. Diabetes Care 2014; 37: 1069-75. 